MATEC Web of Conferences 26,04005 (2015)

DOI: $10.1051 /$ matecconf/ 20152604005

(C) Owned by the authors, published by EDP Sciences, 2015

\title{
Design of a Backdrivable Triglide Robot
}

\author{
Natthapong Angsupasirikul ${ }^{1, a}$, Ratchatin Chancharoen ${ }^{1, b}$ \\ ${ }^{1}$ Chulalongkorn University, Thailand
}

\begin{abstract}
The paper presents a novel Triglide robot that gives low inertia and low friction, back-drivability, no backlash, but stiffness in vertical direction. These features give the robot to be a good candidate as a collaborative assembly robot. A brief kinematics is also included. Compared to the other three translational degrees of freedom (DOF) parallel robots, the proposed Triglide is easier to construct and gives a higher stiffness in vertical direction. Its forward kinematics is also easier compared to those robots. Three experiments are used to demonstrate the capability of the robot including (1) Cascade Step response, (2) Record and Playback with a virtual wall and (3) Force control of the back-drivable Triglide.
\end{abstract}

\section{Introduction}

The history of robots begins in 1920s. In the beginning, it was the machine that assists human doing their tedious and heavy duty. Until the late 1960s, the first industrial robot has been prevailed. Typically, a robot is a set of links, which are connected, by a set of joints. One link must be fixed to a stationary base which is thus called base and another link, called end effecter, has arbitrary motion in space. There are rigid links and joints that connect the base to the end effector. If links and joints are connected in serial chain, then it is called a serial robot. Most of industrial robots today are serial robots. Because of the serial chain, the transmitted force passes through every links and joints and thus the robot structure must be very strong and rigid such that excellent accuracy is achieved.

In contrast, there is another configuration of robot, called parallel robot, which has attracted researcher in the past two decades. The rigid links and joints that connect the base to the end effector are not in a single chain but in parallel kinematic chains. All the actuators are designed to sit on the base and control the motion of the end effector through parallel chains. In this way, the inertia of the robot is relatively low with a higher stiffness and a better positioning accuracy [1]. The summary of comparative features between the serial and parallel robots is shown in Table 1.

Table 1 Comparative features of serial and parallel manipulator

\begin{tabular}{|c|c|c|c|}
\hline Features & Serial Robot & Parallel Robot & Triglide Robot \\
\hline Workspace & Large & Small & Smaller \\
\hline Stiffness & Low & High & $\begin{array}{l}\text { High in vertical } \\
\text { direction }\end{array}$ \\
\hline Inertia & High & \multicolumn{2}{|c|}{ Low } \\
\hline Structure & Simple & Complex & Intermediate \\
\hline Accuracy & Accumulated & \multicolumn{2}{|c|}{ Error Average Out } \\
\hline Motion & Slower & \multicolumn{2}{|c|}{ Faster Speed } \\
\hline Kinematic & IK is complicated & FK is complicated & FK is simpler \\
\hline Control & Simpler & \multicolumn{2}{|c|}{ Complicated } \\
\hline Design Complexity & Low & High & Medium \\
\hline
\end{tabular}

In general, a parallel robot is designed to be symmetrical and the DOF is equal to number of kinematic chains. In this project, we design a parallel robot with three translational DOF. A three translational DOF parallel robot is the most basic parallel mechanism and is in our interested for academic purpose. This robot is also the best candidate for pick and place and

\footnotetext{
a Natthapong.A@Outlook.com ${ }^{\mathrm{b}}$ Ratchatin.C@chula.ac.th
}

machining operation as well. The most famous robot with three translational DOF is a Delta robot [2] that was introduced by Professor Raymond Clavel at École Polytechnique Fédérale de Lausanne. The structure of a Delta robot is designed to be the spatial mechanic that consists of three kinematic closed chains connecting two platforms, which have 120 degree of angle of each. A 
Delta robot has special characteristic and low-mass links. Because of this the robot can reach a huge acceleration up to 50 times of the gravity in experiment and $15 \mathrm{~g}$ in industry. There is also a similar configuration called a Star robot $[3,4]$ that gives three translational DOF. The difference is that the Star robot actuator is prismatic while the delta is revolute.

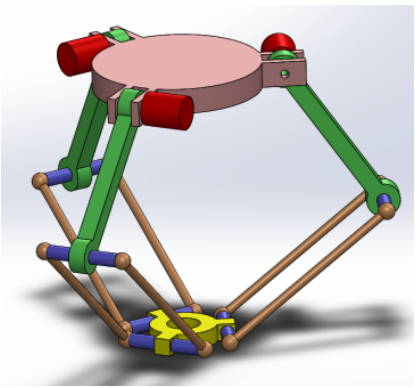

a) A Delta robot

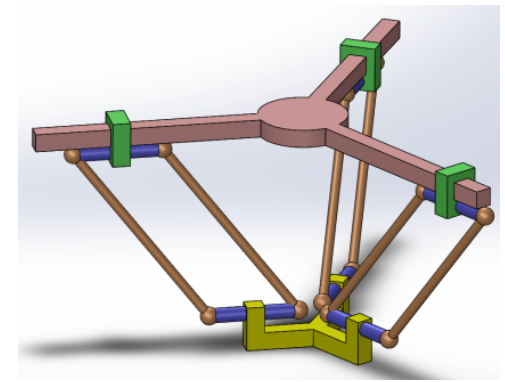

b) A Star robot

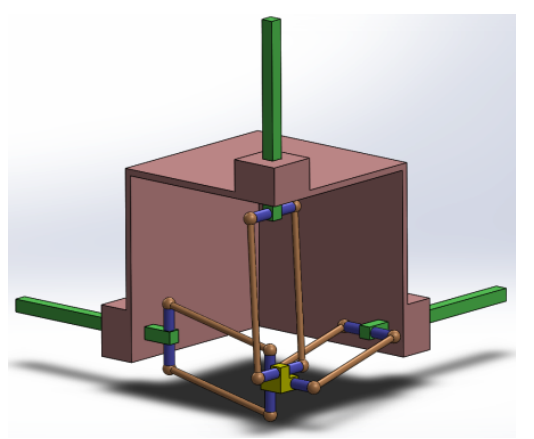

c) An Orthoglide robot

Figure 1. Various Configurations of a three translational DOF parallel robot

In the project, the Triglide robot [6] is designed as a cooperative robot. The cable transmission is used to give the robot a back-drivable capability. The paper addresses several benefits of this configuration with its performance is experimentally demonstrated. One of the main advantages are that it gives good motion characteristic in horizontal plane and good stiffness in vertical direction. This is good for pick and place or drilling application.

\section{A Backdrivable Triglide Robot}

A Triglide robot consists of an end plate, a base, and three limbs with identical kinematic structure. The limbs are parallel mechanisms that constrain the end plate such that it parallel to the base at all time. The limbs connect to sliders those sit on the base which are independently
Another interesting three translational DOF parallel robot is an Orthoglide robot [5] which is developed for high speed machining application. The main advantages of this robot are its good homogenous kinetostatic performance and its workspace that suits to its application.

driven by permanent magnet DC motors with cable transmissions. In this way, the robot is amazingly backdrivable. The torques from the motors can be transmitted to the end plate via low inertia rigid link. Thus, the robot is very rigid with very low inertia. In total, there are eleven links, twelve spherical joints, and three active rotational joints that are driven by three motors.

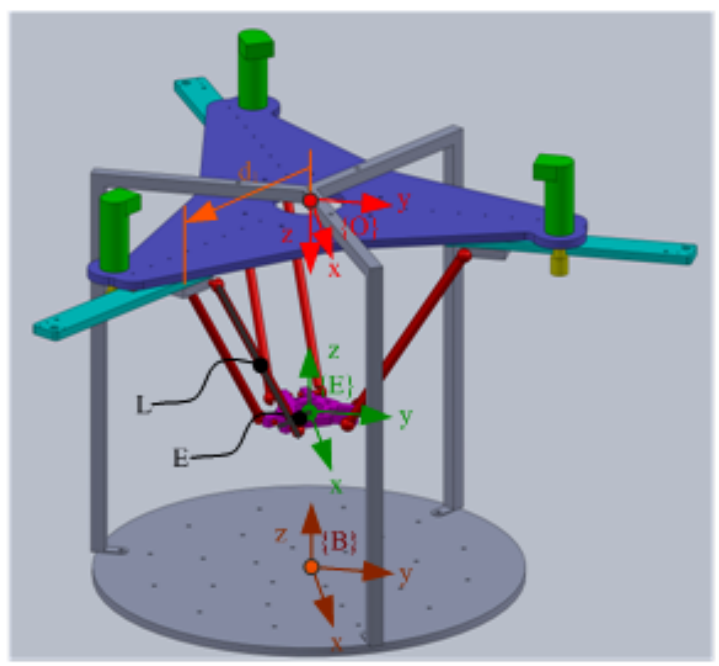

a) CAD model

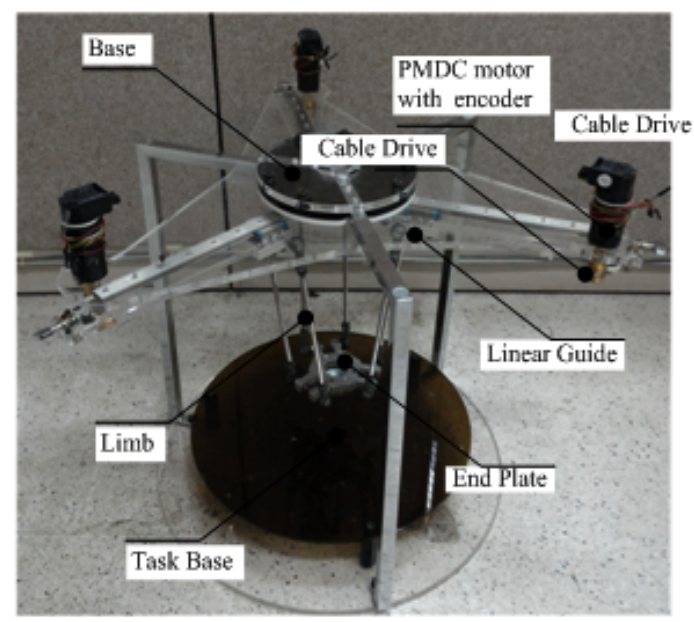

b) Built model

Figure 2. The model of the back-drivable Triglide robot

\section{Kinematics of the Triglide Robot}

In term of kinematics, there are eleven links, twelve spherical joints, and three active slider joints which are driven by three motors. Compared to a serial link manipulator, the kinematics solution of a Triglide is more complicated since it involves twelve passive spherical joints which their positions must be eliminated prior to map the encoder's positions to the end plate's position. In 
this project, the graphical technique is used to develop forward and inverse kinematics of the Triglide robot. The simplified solution is as follows.

\section{Forward Kinematics}

J position $=[\mathrm{d} 1, \mathrm{~d} 2, \mathrm{~d} 3]$;

$\overrightarrow{\mathrm{P} 1}=[0, \mathrm{~d} 1,0] ; \quad \mathrm{P} 2=[\mathrm{d} 2 * \cos (\mathrm{pi} / 6),-\mathrm{d} 2 * \sin (\mathrm{pi} / 6), 0] ; \quad \mathrm{P} 3=[-$ $\mathrm{d} 3 * \cos (\mathrm{pi} / 6),-\mathrm{d} 3 * \sin (\mathrm{pi} / 6), 0]$;

$\mathrm{P} 21=\mathrm{P} 2-\mathrm{P} 1 ; \mathrm{P} 31=\mathrm{P} 3-\mathrm{P} 1 ; \mathrm{n}=\operatorname{cross}(\mathrm{P} 21, \mathrm{P} 31)$;

$\mathrm{P} 0=\operatorname{cross}(\operatorname{dot}(\mathrm{P} 21, \mathrm{P} 21) * \mathrm{P} 31-$

$(\operatorname{dot}(\mathrm{P} 31, \mathrm{P} 31) * \mathrm{P} 21), \mathrm{n}) /(2 * \operatorname{dot}(\mathrm{n}, \mathrm{n}))+\mathrm{P} 1$;

$\mathrm{t}=\operatorname{sqrt}((\mathrm{L} * \mathrm{~L}-\operatorname{dot}(\mathrm{P} 0-\mathrm{P} 1, \mathrm{P} 0-\mathrm{P} 1)) / \operatorname{dot}(\mathrm{n}, \mathrm{n}))$;

Robot_position $=\mathrm{P} 0+\mathrm{n} * \mathrm{t}+\left[\begin{array}{ll}0 & 0 \mathrm{~L}\end{array}\right]$;

where $\mathrm{d} 1, \mathrm{~d} 2$, and $\mathrm{d} 3$ are the positions of the robot joint \#1, \#2, and \#3 respectively.

$[\mathrm{X} Y \mathrm{Z}]$ is the Cartesian position of the end plate. $\operatorname{cross}(. ., .$.$) is the cross product of two vectors.$ $\operatorname{dot}(. ., .$.$) is the dot product of two vectors.$ $\operatorname{sqrt}(.$.$) is the square root function.$

\section{Inverse Kinematics}

Robot_position $=[\mathrm{X}, \mathrm{Y}, \mathrm{Z}]$

$$
\begin{aligned}
& \mathrm{A}=\left[\mathrm{X}, \quad-\mathrm{X}^{*} \sin (\mathrm{pi} / 6)-\mathrm{Y}^{*} \cos (\mathrm{pi} / 6),\right. \\
& \left.\mathrm{X}^{*} \sin (\mathrm{pi} / 6)+\mathrm{Y}^{*} \cos (\mathrm{pi} / 6)\right] ; \\
& \mathrm{B}=\left[\mathrm{Y}, \quad \mathrm{X}^{*} \cos (\mathrm{pi} / 6)-\mathrm{Y}^{*} \sin (\mathrm{pi} / 6), \quad-\mathrm{X}^{*} \cos (\mathrm{pi} / 6)-\right. \\
& \left.\mathrm{Y}^{*} \sin (\mathrm{pi} / 6)\right] ; \\
& \mathrm{J}[\mathrm{x}]=\operatorname{sqrt}\left(\mathrm{L} * \mathrm{~L}-\mathrm{A}[\mathrm{x}]^{*} \mathrm{~A}[\mathrm{x}]-(\mathrm{L}-\mathrm{Z}) *(\mathrm{~L}-\mathrm{Z})\right)+\mathrm{B}[\mathrm{x}]+\mathrm{E} ;
\end{aligned}
$$

Table 2 The Triglide robot's parameters

\begin{tabular}{|l|r|r|}
\hline Parameter & Value & unit \\
\hline L Limb length & 250.00 & $\mathrm{~mm}$ \\
\hline E End plate radius & 50.00 & $\mathrm{~mm}$ \\
\hline J minimum & 79.25 & $\mathrm{~mm}$ \\
\hline J maximum & 245.25 & $\mathrm{~mm}$ \\
\hline J resolution & 0.1431 & $\mathrm{~mm}$ \\
\hline
\end{tabular}

\section{Control Technique}

The Triglide robot in the project is driven by three permanent magnet DC motor with 100 PPR. The 86duino ONE microcontroller board is used as the main processor, which captures all the three encoders with $\mathrm{X} 4$ decoder, solves inverse kinematics at runtime, applies the control law, and sends the control efforts to the three motors. The VHN 5019 motor drivers are used to drive the motor. The 24 volts power supply is used to power the motor driver. The motors are driven in voltage mode but the currents flowed though the motors are monitored.

The proposed inverse kinematics is used to solve for the three joint's coordinates which will be used as the joint's reference positions at every servo loop. The proportional controller is used to control the three joints simultaneously to the references at $1 \mathrm{kHz}$ servoing rate. The Pulse-Width Modulation (PWM) is operated at 20 $\mathrm{kHz}$ and the power supply to the motor driver is at 24 volts.

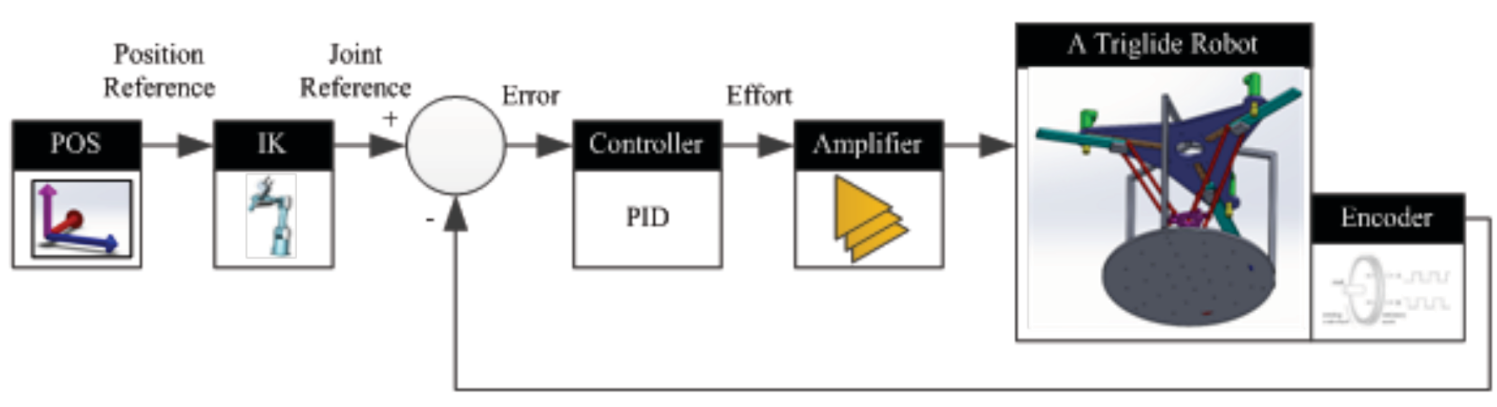

Figure 3. Control Diagram

\section{Experimental Results}

Three experiments are used to demonstrate the capability and performance of the proposed back-drivable Triglide robot.

\section{Experiment I: Cascade Step response}

The cascade Step response is used to investigate the performance of each joint controller and also interference to the other two joints. The first joint is commanded to follow a step position at 175 and $50 \mathrm{~mm}$, back and forth, with 5 second interval. The other two joints are also commanded to follow a similar step trajectory, but with
1.667 and 3.333 second time shift respectively. The proposed Triglide robot with cable drive has a very low friction and its damping is quite low. There are noticeable interferences among the three joints. When the joint is sturdily moving, the other two joints shake and there are current flows through these motors. 


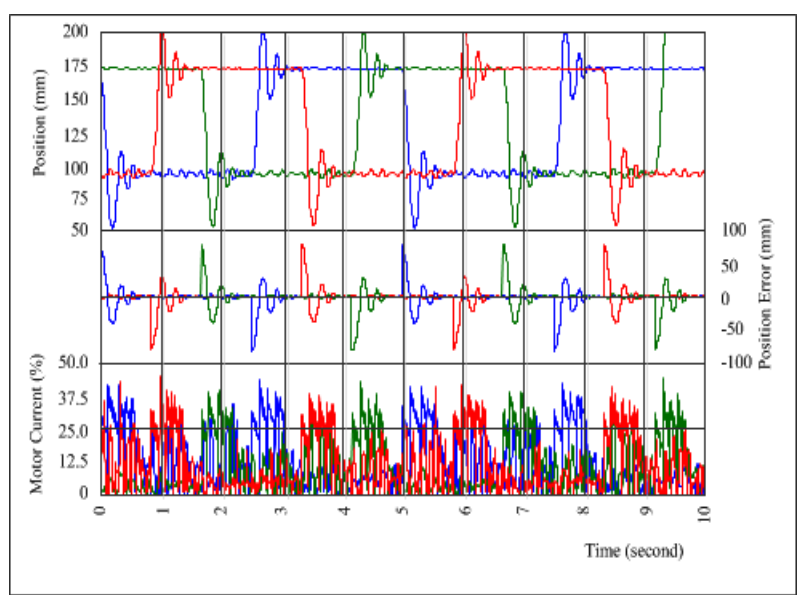

Figure 4. Cascade Step response

Experiment II: Record and Playback with a virtual wall

There capability of the proposed backdrivable Triglide robot is demonstrated in this experiment. The robot is back-drivable, thus a user is able to grab the robot's end plate and move it around its workspace. The trajectory is recorded and shown with RED line in Fig. 5. Low inertia is noticed during the record mode. The robot is then commanded to follow the record trajectory. The playback trajectory, shown in BLUE, is plotted and overlaid on the record trajectory. In this experiment, the virtual wall [7] in horizontal plane at $\mathrm{z}=30 \mathrm{~mm}$ is also activated. The robot controller maintains the end plate on the plane at $\mathrm{z}=30 \mathrm{~mm}$ while the motion in this plane is free which a user can move it with low resistant. In this design, a stiff virtual wall can be implemented and the robot gives good accuracy despite it is back-drivable. The Triglide robot is very stiff in vertical direction and less stiff in horizontal plane.

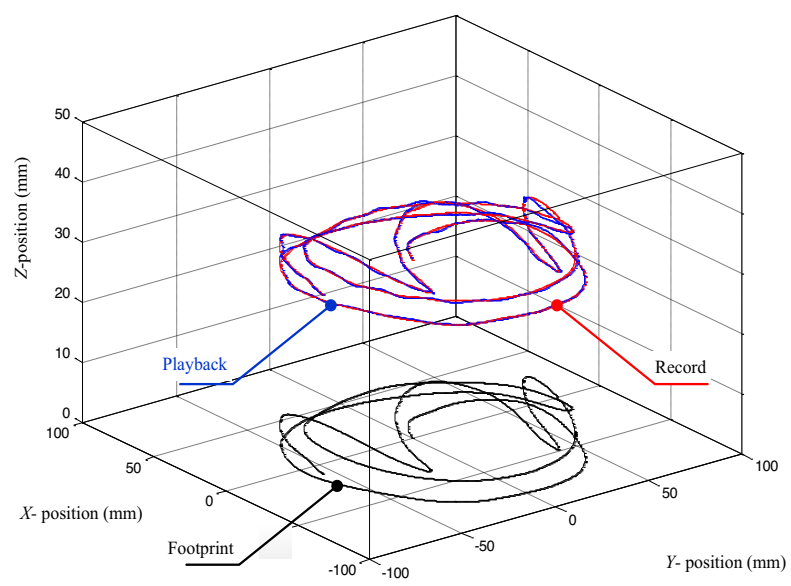

Figure 5. Performance in Record and Playback mode

Experiment III: Force control of the back-drivable Triglide.

The three-axis force at the end plate can be measured by the current flow through the three permanent magnet DC motors. Thus, we can control the touching force while the robot moves as demonstrated in Fig. 6. In the experiment, the robot is commanded to travel along a circular trajectory in horizontal plane while keeping the touching force at zero. The impedance control [8] is used to control force during the motion. The proposed robot use control effort to estimate force thus, requires no additional hardware to sense force.

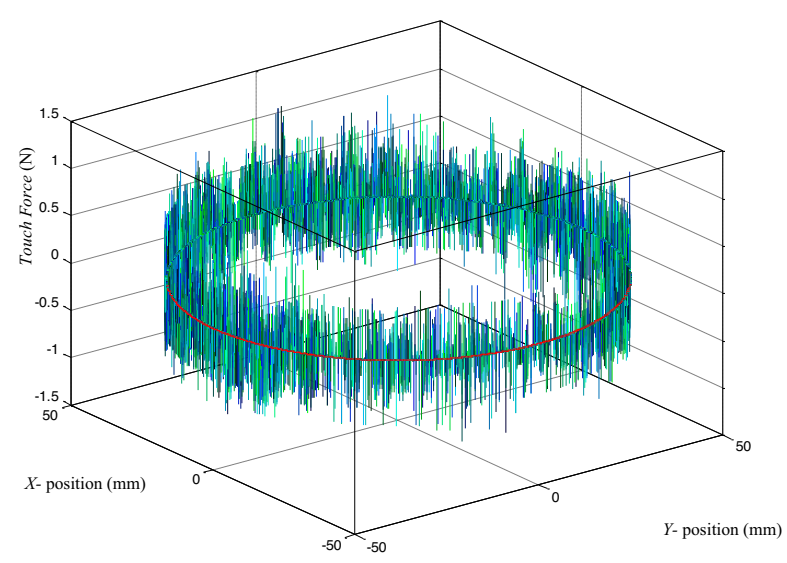

Figure 6. Force control in circular trajectory

\section{Summary}

The proposed back-drivable Triglide robot is a collaborative robot that is designed for three translational DOF task. The robot gives low inertia and friction and no backlash. The robot is back-drivable and thus a human operator can guide the robot's end plate to program the robot's motion. The proposed robot is very stiff in vertical direction but less stiff in the horizontal plane. Thus, it is a good candidate for an assembly robot. Moreover, the touching force can be determined from the current flow through the three motors. This allow us to control the touching force without additional hardware.

\section{References}

1. Kok-Meng Lee, Shah, and D.K. (1988). Kinematic analysis of a three-degrees-of-freedom in-parallel actuated manipulator, IEEE Journal of Robotics and Automation Vol.4, p.354

2. Olsson, A. (2009). Modeling and Control of a Delta3 robot, Lund University, Sweden.

3. Sparacino, F., Herve, J.M. (1993). Synthesis of parallel manipulators using Lie-groups Y-STAR and H-ROBOT, submitted to Advance Robotics conference.

4. Yuehua Wan, Guan Wang, Shiming Ji, Zhongfei Wang, and Jun Liu (2008). A Y-Star Robot: Optimal Design for the Specified Workspace, submitted to Conference on Computer Science and Software Engineering.

5. A. Pashkevich, D. Chablat, and P. Wenger (2006). Kinematics and workspace analysis of a three-axis parallel manipulator: the Orthoglide. Robotica, 24, p. $39-49$

6. Stan S., Manic M., Maties V., and Balan R. (2008). Evolutionary approach to optimal design of 3 DOF translation exoskeleton and medical parallel robots, Conference on Human System Interactions, p.720. 
7. Colgate J.E., Grafing P.R., and Stanley M.C. (1993). Implementation of stiff virtual walls in forcereflection interfaces, Virtual Reality Annual International Symposium, p.202
8. Hogan, N. (1985), Impedance control: an approach to manipulation. Journal of Dynamic Systems, Measurement and Control, Transactions of the ASME 107 (1), p. 1-24. 